\title{
Driver State Alert Control using Head Shoulder Inclination and Facial Landmarks
}

\author{
Ashitha Ebrahim, Fathimathul Harshima P. T., Aby Abahai T.
}

\begin{abstract}
Driver sleepiness is one in all the most important causes of most of the accidents within the world. Detecting the driver's eye weariness is the simplest way for detecting the somnolence of the driver. The prevailing systems in the literature cannot discover sleepiness in folks having lagophthalmos (condition that forestalls eyes from closing utterly) and monocular vision (person with one-eyed or sight loss in one eye). To solve this downside a Driver State Alert Control system is projected which makes the use of head-shoulder inclination, face detection, eye detection, emotion recognition, eye openness estimation and blink counts for detecting the sleepiness and collision liability associated with robust emotional factors. The projected framework endlessly analyses the head shoulder inclination and facial lineaments of the driver to alert the driver by activating the alarm once he/she is drowsy or showing emotion unstable to drive. The typical separation between upper eyelid and lower eyelid of adults suffering from lagophthalmos is about 1-5mm. The EAR (Eye Aspect Ratio) is calculated supporting this separately for each eye. Thus, the proposed technique can be used for folks with lagophthalmos and monocular vision. Also, the entities don't solely rely on blink count to ascertain the sleepiness, collision risks related to robust emotional factors are considered too. The projected system which is enforced with one camera view on open $C V$ and raspberry pi setting illustrates the systems good efficiency in particulars of authentic sleep identification results and thus reduces road mishaps. It is easy to place in any type of vehicle and price effective too.
\end{abstract}

Keywords: Lagophthalmos, EAR, Open CV, Raspberry pi

\section{INTRODUCTION}

$\mathrm{D}$ river drowsiness is one of the significant reasons for the majority of the mishaps in the world [1]. Recognizing the driver's eye tiredness is the simplest path for estimating the laziness and the feelings of the driver. Here displayed a novel vision framework for driver checking. It is intended for simple and adaptable arrangement of substance in any kind of vehicles with no additional equipment cost other than for raspberry pi establishment is required. In most existing

Revised Manuscript Received on February 05, 2020

* Correspondence Author

Ashitha Ebrahim*, Department of Computer Science and Engineering, Mar Athanasius College of Engineering, Kothamangalam, India. Email: achuashitha5@gmail.com

Fathimathul Harshima P.T., Department of Computer Science and Engineering, Mar Athanasius College of Engineering, Kothamangalam, India. Email: harshima123@gmail.com

Prof. Aby Abahai T., Department of Computer Science and Engineering, Mar Athanasius College of Engineering, Kothamangalam, India. Email: abytom@gmail.com

(C) The Authors. Published by Blue Eyes Intelligence Engineering and Sciences Publication (BEIESP). This is an open access article under the CC BY-NC-ND license (http://creativecommons.org/licenses/by-nc-nd/4.0/) vehicles, there are as of now numerous arch cameras introduced for security purposes. One is mounted in the upper right or upper-left situation concerning the driver to record the driver's conduct on obligation. Since this camera is first introduced for the account of driving conduct, a wide-see vault camera is utilized to catch the unmistakable chest area of the driver.

To acquire a precise and strong gauge of eye receptiveness, a novel combination calculation proposed which adaptively coordinates the consequences of eye transparency estimations on the multi-model eye recognitions for both eyes [2]. In view of the imaginative methods, the framework accomplishes strong execution on the difficult situations where the current methodologies frequently come up short. It applies to low-goals face pictures caught from a survey point to the driver's face with the goal that it can share a wide-see camera mounted for driver's full-body conduct observing. The innovative commitments can be outlined as follows:

- An epic diagram for vision-based weakness recognition which incorporates head-shoulder location, eye transparency estimation, combination, multi-present face discovery, multi-model eye identification and PERCLOS estimation for weariness location.

- A Boost learning calculation to procure a mapping from a low-goal eye picture to a steady degree of eye transparency

- A Computational calculation to acquire an exact and hearty eye receptiveness gauge dependent on versatile mix on multi-model eye recognitions on the two eyes.

This paper hopes to develop an instance of a tired driver cautioning framework [3]. Our whole focus and obsession will be put on organizing the structure that will decisively control the open and close circumstance of the driver's eye ceaselessly. By ceaselessly checking the eyes it might be seen that to keep up a vital good ways from setbacks the signs of driver depletion can be recognized early enough. This spotting should be conceivable using a progression of pictures of eyes similarly as head and face improvement. The movement of eye improvements and its edges for the distinctive evidence will be used. Contraptions to distinguish when drivers are falling in a significant rest and to offer alarms to alert them of the risk or even control the vehicles improvement have been the ward upon a lot of inventive work. Weariness is a troublesome issue results distinctive road setbacks consistently. It isn't at present possible to figure the particular number of rest related accidents in perspective on the difficulties in recognizing whether exhaustion was a segment. 


\section{Driver State Alert Control using Head Shoulder Inclination and Facial Landmarks}

Whether or not you are uneasy peaceful or hot-tempered your characteristics sway the way where you drive. Do whatever it takes not to let your sentiments debilitate with safe driving [4]. Use all the consideration pragmatic knowledge practical insight and safe driving rules that you can. Phenomenal sentiments of any kind whether or not negative or positive increase the likelihood that the driver isn't giving close consideration to the driving scene and the cars around. It just takes one minute to miss a notification sign or to miss a gander at danger out of the side of your eye. In light of your disposition you may in like manner face more driving difficulties than you normally would when you are free calm and alert. Disquiet stress fear and other eager points of view can and will hurt your driving limit. Interference is the primary wellspring of basic effects. Stress and exhaustion are critical wellsprings of obstruction. Remember the importance of recognition and sharpness to the driving condition can't be over pushed.

An epic vision framework is proposed to manage these difficulties, which incorporates chest area discovery, face recognition, eye identification, eye transparency estimation, combination, and manifestation measure estimation. We propose a complex learning calculation which can take in a mapping from a low-goals eye picture (e.g., $32 \times 24$ pixels) to a 1-dimensional persistent degree of eye-receptiveness.

\section{RELATED WORKS}

Evaluators generally issue their appraisals as a piece of the continuous learning technique and there's a developing procedures. The human exertion required for the evaluation is exceptionally high. It relies upon a few factors for example information on the instructor application-level comprehension of the educator criteria of the checking and time dispensed. Any way it expends exorbitant endeavor's and sets aside immense effort for the finishing of the total assessment check and distributing of the subsequent procedure.

\section{A. Drowsiness Detection Based on Visual Features}

Driver languor is a typical wellspring of lethal auto collisions. Right now driver help framework with a double power plan is created; it endeavor's to complete at the same time the security sway of the vehicle and recognition of the driver's condition [5]. The structure actualizes in unlimited authority in the event of path flight and permit the driver the likelihood to uninhibitedly make the strides required. In the event that the driver prematurely ends to apparatus the guiding activity required inside a restricted time the help framework reasons that the drivers sympathetic of the given situation is wrong and completes the rest of the control. We utilized a driving test system outfitted with the help framework to investigate the adequacy of distinguishing driver laziness and forestalling path flight mishaps

\section{B. Estimating vigilance level by using EEG and ECG signals}

It was focused to figure the readiness level by utilizing both EEG and EMG signals for developing the rightness of the assessment rate [6]. In our framework EMG and EEG signals were gained from 30 subjects. In the information blend writing on anyway best to coordinate and formalize these

arrange EEG signals were disengaged to its sub-groups utilizing wavelet change for efficient separation and jaw EEG was utilized to affirm and expel the movement relics. the coins in EEG and EMG were resolved while the advancement from attentiveness to rest by utilizing developed counterfeit neural systems preparing and testing informational index contains and the force thickness of EMG signals and sub-joined parts of EEG were placed for preparing and testing the model which gives three answers for the observation level of the subject: rest sluggish and alert.

\section{Physiological Approaches}

The physiological signs from a body like partner degree encephalogram EEG for mind action Electrooculogram (EOG) for eye development and cardiogram ECG for beat rate territory unit assessed to watch driver somnolence [7]. Late investigations show that the methodologies exploitation physiological signals particularly the electroencephalogram sign can do higher responsibleness and precision of driver sleepiness location contrasted with various procedures. Anyway the nosy idea of measure physiological signs will upset driving especially for delayed driving periods.

\section{Vehicle Based Approaches}

Gather signal knowledge from sensors in vehicles to pass judgment on the driver's performance [8]. These ways screen the varieties of hand wheel point path position speed quickening and braking to anticipate driver weakness. It's advantageous to accumulate vehicle signals. Be that as it may these methodologies could be too delayed to even consider finding driver fatigue.

\section{E. Behavior Based Approaches}

Rely upon vision investigation to watch driver's conduct, together with eye-conclusion, eye-squinting, yawning, head present, hand motion, and so forth., through a camera coordinated to the driver's face[9][10]. The intention power is cautioned if an impermanent state manifestation is distinguished. The vision-put together frameworks with respect to conduct examination square measure connecting with to car ventures since \{they square measure they're\} non-meddlesome to the thought process power and in this way the measures are successful and solid to foresee driver weakness. A sluggish driver shows assortment of side effects, together with visit eye-conclusion, fast and steady flickering, underact or swinging head, and continuous yawning. The vast majority of the predominant frameworks might want the establishment of a camera legitimately toward the driver's face to catch high-goals face photographs, and several of them utilize explicitly structured Infra-Red (IR) cameras or stereo cameras. The vision calculations square measure intended for high-goals front-see face and eye pictures [11] [12]. This design isn't material for and gigantic vehicles. A vehicle to a great extent includes a mammoth front glass window to let the thought process power have a wide-field read of the scene for safe driving since it's bounteous more extensive than autos. Putting a camera on the front glass window isn't reasonable, which conjointly obstructs the driver's read. Physiological signals (particularly the graphical record signal) can do higher dependableness and precision of driver transitory state location contrasted with elective methodologies. 
Be that as it may, the nosy idea of mensuration physiological signs will upset driving, especially for delayed driving periods.

\section{F. Haar Cascade Classifier}

The framework utilizes the Haar principally based course classifier for eye attaching and HOG-SVM blend for wink discovery. When squint recognition the PERCLOS worth was calculated [13].If the PERCLOS esteem was over 6 seconds the individual was recognized as sluggish what's more, else he was named as dynamic. The framework was any approved by correlation results with the perceptions of a human rate. The framework gave results with exactness similar to that of a person's onlooker. The presented framework performs well beneath conventional lighting conditions and conventional goals. The strategy is nonintrusive and therefore simple. It doesn't need any exceptional equipment separated from a standard web camera. This makes the framework fitting to be implemented in personal computers, cell phones and afterward on. This system will be used in a decent style of utilizations like driver readiness mensuration, exuberance location, fixation mensuration, a live of mindfulness, and so forth.

\section{PROPOSED SYSTEM}

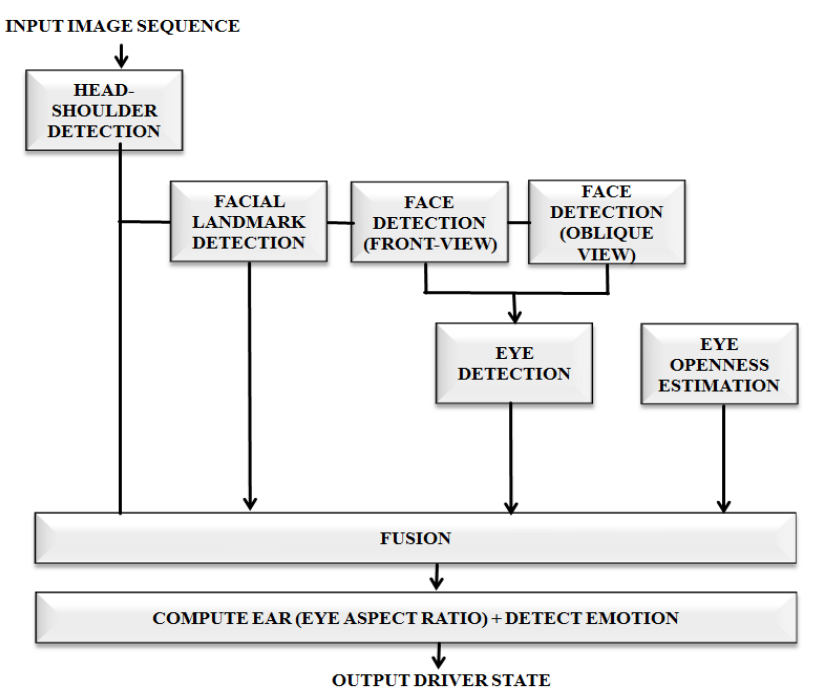

Fig. 1.Block Diagram

The purpose of driver state alert control system is to accomplish fatigue detection along with emotional state detection of the driver. We place dash camera inside the car to monitor the face, the head-shoulder inclination and also the eye movement of the driver to ascertain that the driver is in condition to drive or not. Here we can determine if the eyes are open or closed. Consequently, a warning signal should be generated.

The main precedent of this system is that it must be highly non-presumptuous and should start when the ignition is turned on without having the driver conscript the system. The driver should be liable for providing any feedback to the system. The system must also operate nevertheless of the texture, color of the face, changes in light, shadows, reflections etc.

The Driver state alert control system consists of:

\section{A. Head Shoulder Detection}

To recognize the occupancy of a driver and find generally the situation of the driver's head, a Haar course classifier is prepared for human head-shoulder recognition. Raspberry pi 3 procedures the video outline by edge to distinguish the existence of people by utilizing the aforesaid prepared course classifier for head-shoulder discovery. This procedure gives the check of people present inside the scene.

\section{B. Facial Landmark Detection}

In this module, we detect a front-view face or an oblique-view face within the region of the head. 68-point facial landmark Haar cascades is employed for this. The course technique is a lot quicker than HOG and Linear SVM detection algorithms. Here, we can detect human facial landmarks and use them further in ascertaining EAR and identifying feeling of the driver.

\section{Eye Detection and Eye Openness Estimation}

To locate the potential eye positions and scales in the image is applied to the located eyes from facial points which are indicated for eyes. There are 6 points for each left and right eyes as shown in fig below:

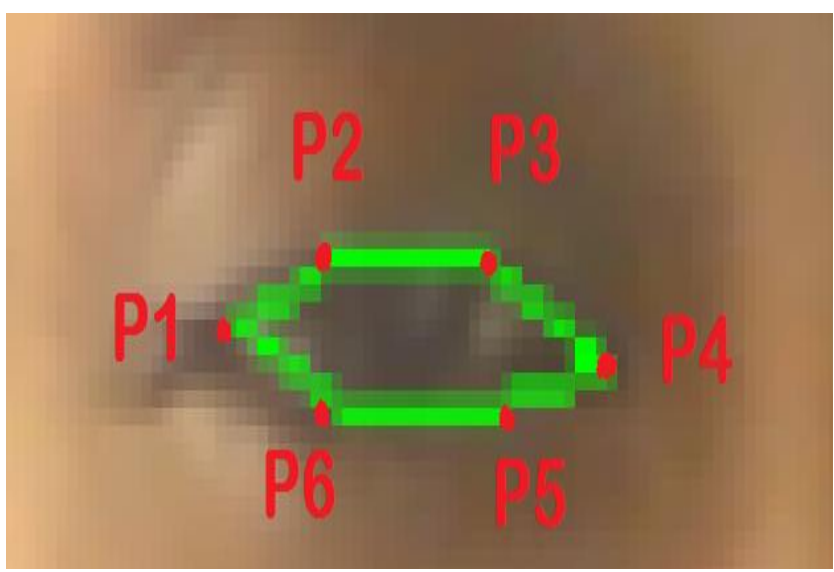

Fig. 1.Eye Openness Estimation

\section{Fusion Operation}

To obtain an accurate and robust estimate of driver's eye openness based on adaptive integration on multi-model eye detections for both eyes

\section{E. Measure EAR and Identify Emotion}

The prediction of emotion from 68-point facial landmark is done using Support Vector Machine (SVM). To train an AI calculation (we will utilize a linear SVM). The main activity is discover approaches to change these 68-facial landmark dots overlaid all over into traits to bolster the classifier. These traits are small amounts of data that portray the driver state that we are attempting to partition into classifications. We train the system for two emotions ie. Anger and sad only. On the off chance that both of these characteristics appeared by driver, then an alert given to driver because emotional well-being of driver is also vital while driving. 


\section{Driver State Alert Control using Head Shoulder Inclination and Facial Landmarks}

One bit of leeway of the SVM model we use is that it is probabilistic. This implies it doles out prospects to every apportionment it has been equipped for (and you can get these probabilities on the off chance that you set the 'probability' flag to True). In this way, for instance, a solitary picture may be "happy" with 85\% possibility, "angry" with "10\% possibility, and so forth.

\section{F. Eye Detection and Eye Openness Estimation}

To locate the potential eye positions and scales in the image is applied to the located eyes from facial points which are indicated for eyes. There are 6 points for each left and right eyes as shown in fig below

The Eye Aspect Ratio is calculated as follows:

leftEAR = eye_aspect_ratio(leftEye)

rightEAR = eye_aspect_ratio(rightEye)

ear $=($ leftEAR + rightEAR) $/ 2.0$

The function eye_aspect_ratio calculated as:

def eye_aspect_ratio(eye):

$\mathrm{A}=$ dist.euclidean(eye[2], eye[6])

$\mathrm{B}=$ dist.euclidean(eye[3], eye[5])

$\mathrm{C}=$ dist.euclidean(eye[1], eye[4])

ear $=(A+B) /(2.0 * C)$

\section{G. Alert Driver}

Traffic HAT used to create the alarm that will sound if a driver/user gets tired.

\section{IMPLEMENTATION}

The driver state alert control is contrived using the Raspberry pi $\mathrm{B}+$ model in conjunction with a dash camera which can be ordinarily installed in any vehicle. Moreover, a portable HDMI display (optional) can be connected which can consequently add interactive visual capability for Raspberry pi. A VNC server is about over here to attach the laptop display to the raspberry pi via coax cable in reverse to the HDMI displaying the results on the Raspberry Pi of our optimized driver drowsiness detection algorithm. Implement dlib facial landmark modifications so our drowsiness detector is deployed to the Raspberry Pi. Haar cascade face detector of Open CV is employed to detect face of the driver. We use dlib for face detection and facial landmark detection. Out of the 68 facial landmark points 6 points are for each left and right eyes. The imutils package has been imported to dlib in raspberry pi handle the 68-point facial landmark model.

The Traffic HAT used to create the alarm that will sound if a driver/user gets tired or emotion (anger and sadness) is detected.

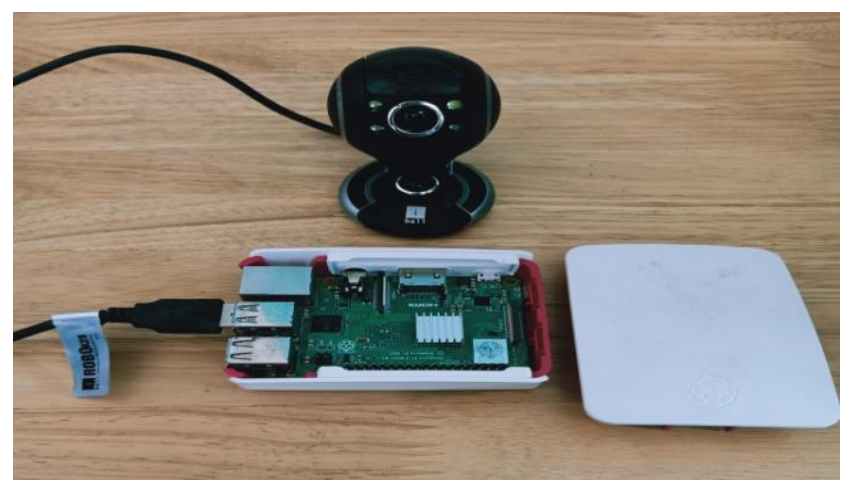

Fig. 2. Experimental Implementation of the conceptual system for the Driver State Alert Control System

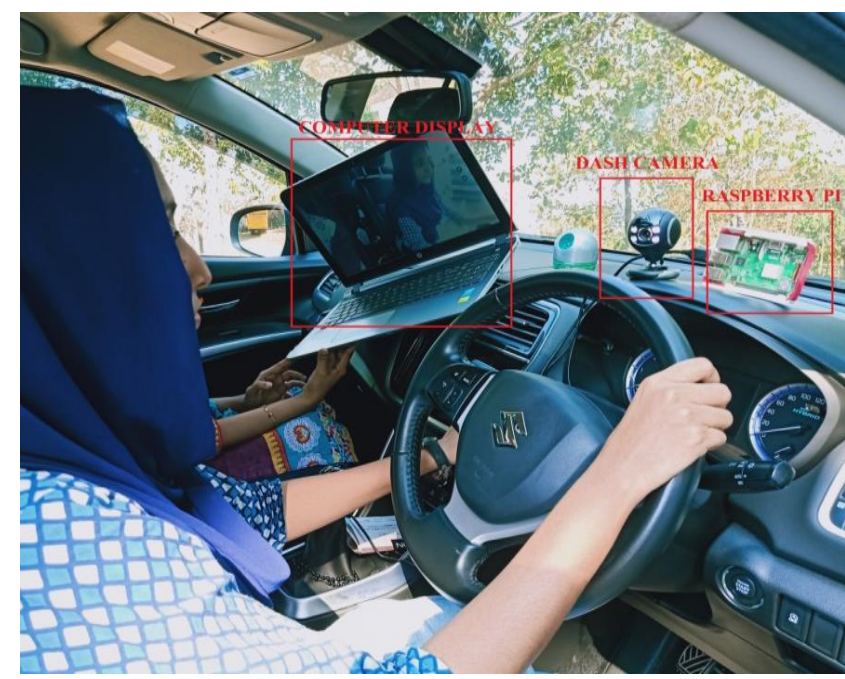

Fig. 3. Configuration of the Proposed Driver State Warning Control System on a prototype car

\section{RESULTS}

Results shows the screenshot of the GUI which displays the Eye Aspect Ratio (EAR) and also displays "DROWSINESS ALERT" if EAR is below the threshold value. The web camera continuously monitor the eye blinking ratio of the driver along with the head-shoulder inclination. Then calculate the PERCULOS value to see if the driver is drowsy or not.
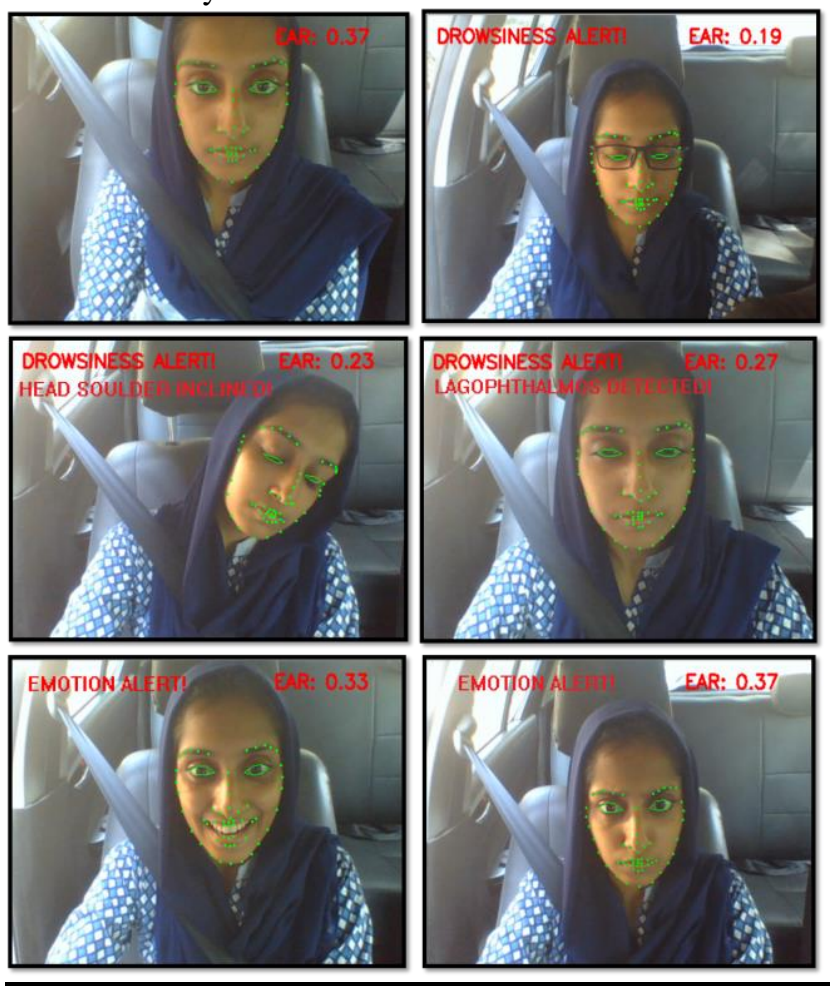

Fig. 4. (a) Screenshot of image, during which eye is open and corresponding EAR is calculated and shown on prime right.

(b) Screenshot of image, within which eye is closed absolutely and sleepiness alert is shown. 
(c) Screenshot of image, in which eye is closed absolutely with head shoulder inclination and sleepiness alert is shown. (d) Screenshot of image that shows eye is closed partly with head shoulder inclination and sleepiness alert is shown.

(e) Screenshot of image, during which emotions (happy and anger respectively) and emotion alert is displayed (f) Screenshot of image, showing sleepiness alert in people with monocular vision (have one eye or sight loss in one eye) (g) Screenshot of image, were sleepiness alert shown in people with abnormal condition (condition that stops eyes from closing completely)

\section{CONCLUSION}

Driver somnolence is a serious danger and chief concern that is observed as animmediate orcontributing motive in maxim um road injuries. Establishing Driver state alert control systemthatactasamonitoring device thatcan provide signals $t$ o the driver when symptoms of lethargy are detected can save you road accidents and thus save lives. The cost of deployment of this proposed system is cheap and potent in alerting the driver state with a fast repose time.

\section{REFERENCES}

1. B. Mandal, L. Li, G. S. Wang and J. Lin, "Towards Detection of Bus Driver Fatigue Based on Robust Visual Analysis of Eye State," in IEEE Transactions on Intelligent Transportation Systems, vol. 18, no. 3, pp.545-557, March 2017.

2. P. Wang and L. Shen, "A method of detecting driver drowsiness state based on multi-features of face," 2012 5th International Congress on Image and Signal Processing, Chongqing, 2012, pp. 1171-1175.

3. D. T. Nhan, T. Q. Bao and T. Q. Dinh, "A study on warning system about drowsy status of driver," 2017 Seventh International Conference on Information Science and Technology (ICIST), Da Nang, 2017, pp. 215-222.

4. Suchitra, Suja P. and S. Tripathi, "Real-time emotion recognition from facial images using Raspberry Pi II," 2016 3rd International Conference on Signal Processing and Integrated Networks (SPIN), Noida, 2016, pp.666-670.

5. Fouzia, R. Roopalakshmi, J. A. Rathod, A. S. Shetty and K. Supriya,M "Driver Drowsiness Detection System Based on Visual Features," 2018 Second International Conference on Inventive Communication and Computational Technologies (ICICCT), Coimbatore, 2018, pp. 1344-1347.

6. R. P. Balandong, R. F. Ahmad, M. N. Mohamad Saad and A. S. Malik, "A Review on EEG-Based Automatic Sleepiness Detection Systems for Driver," in IEEE Access, vol. 6, pp. 22908-22919, 2018.

7. A. Chowdhury, R. Shankaran, M. Kavakli and M. M. Haque, "Sensor Applications and Physiological Features in Drivers' Drowsiness Detection: A Review," in IEEE Sensors Journal, vol. 18, no. 8, pp. 3055-3067, 15 April15, 2018.

8. M. Chakraborty and A. N. H. Aoyon, "Implementation of Computer Vision to detect driver fatigue or drowsiness to reduce the chances of vehicle accident," 2014 International Conference on Electrical Engineering and Information Communication Technology, Dhaka, 2014, pp. 1-5.

9. H. Kang, "Various Approaches for Driver and Driving Behavior Monitoring: A Review," 2013 IEEE International Conference on Computer Vision Workshops, Sydney, NSW, 2013, pp. 616-623.

10. S. Kaplan, M. A. Guvensan, A. G. Yavuz and Y. Karalurt, "Driver Behavior Analysis for Safe Driving: A Survey," in IEEE Transactions on Intelligent Transportation Systems, vol. 16, no. 6, pp. 3017-3032, Dec. 2015.

11. Z. Jie, M. Mahmoud, Q. Stafford-Fraser, P. Robinson, E. Dias and L. Skrypchuk, "Analysis of Yawning Behaviour in Spontaneous Expressions of Drowsy Drivers," 2018 13th IEEE International Conference on Automatic Face Gesture Recognition (FG 2018), Xi'an, 2018, pp. 571576.

12. A. Bartra et al., "A feasability study of drowsiness detection using driving behaviour parameters," 2012 IEEE Intelligent Vehicles Symposium, Alcala de Henares, 2012, pp. 111116.

13. L. Pauly and D. Sankar, "Detection of drowsiness based on HOG features and SVM classifiers," 2015 IEEE International Conference on Research in Computational Intelligence and Communication Networks (ICRCICN), Kolkata, 2015, pp. 181-186.
14. Abbas, Syed Ameer \& Anitha, M. \& Jaini, X.. (2017). Realization of multiple human head detection and direction movement using Raspberry Pi. 1160-1164. 10.1109/WiSPNET.2017.8299946.

15. Kanade, T., Cohn, J. F., \& Tian, Y. (2000). Comprehensive database for facial expression analysis. Proceedings of the Fourth IEEE International Conference on Automatic Face and Gesture Recognition (FG'00), Grenoble, France, 46-53.

\section{AUTHORS PROFILE}

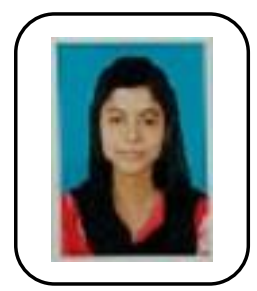

Ashitha Ebrahim received Master of Technology in Computer Science and Engineering from Mar Athanasius College of Engineering affiliated to APJ Abdul Kalam Technological University in 2020 and received Bachelor of Technology in Computer Science and Engineering from Ilahia College of Engineering and Technology in 2018. Her research interest is in Bioinformatics and Data Mining

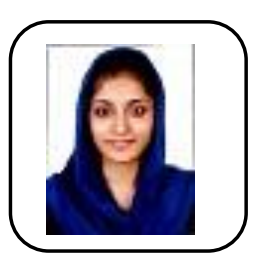

Fathimathul Harshima PT received Master of Technology in Computer Science and Engineering from Mar Athanasius College of Engineering affiliated to APJ Abdul Kalam Technological University in 2020 and received Bachelor of Technology in Computer Science and Engineering from MES College of Engineering Kuttipuram in 2017. Her research interest is in Bioinformatics and

Data Mining.

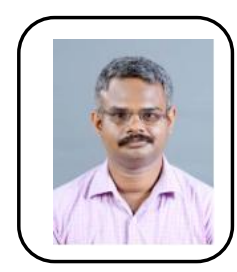

Prof. Aby Abahai $\mathbf{T}$ is currently working as Associate Professor of Computer Science and Engineering department in Mar Athanasius College of Engineering, Kothamangalam, Kerala, India. He received his B-Tech Degree in Computer Science and Engineering in 1999 from Calicut university and M-Tech in Computer Science and Engineering from NITK Surathkal in 2009.He has around 19 years of teaching and research experience in various institutions in India. His research interests include computer design, algorithm analysis and design , system software, theory of computation, artificial intelligence and object oriented programming.

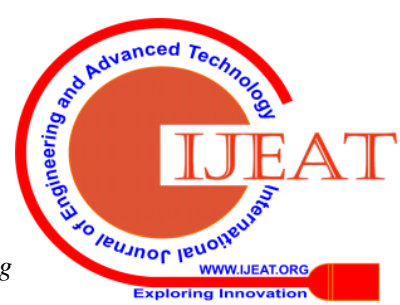

